\title{
Cerebral venous thrombosis: a spectrum of imaging findings
}

\author{
Shuliang $\underline{G e}^{1}$, FRCR, MBBS, Jinhang $\underline{W e n^{1}}$, MBBS, FRCR, Pin Lin $\underline{K e} i^{1}, M B C h B, F R C R$
}

\begin{abstract}
INTRODUCTION
Cerebral venous thrombosis (CVT) is uncommon compared to cerebral arterial disease, and its initial clinical manifestations are often non-specific. The most common presenting symptom is headache, while other clinical manifestations include focal neurological deficit, seizures, nausea and/or vomiting, and altered mental status. ${ }^{(1)}$ Unenhanced head computed tomography (CT) is usually the first-line investigation of choice for these patients. However, the most striking findings on imaging can mimic other pathologies, leading to delayed or wrong diagnoses. As such, a good understanding of the pathophysiology, anatomy and myriad imaging findings of CVT is pivotal in making a timely and accurate diagnosis. An early diagnosis enables early institution of appropriate treatment, resulting in significantly improved patient morbidity and mortality. ${ }^{(2)}$ In this article, we illustrate a wide spectrum of CVT cases.
\end{abstract}

\section{PATHOPHYSIOLOGY AND RISK FACTORS}

Two main mechanisms that contribute to the clinical presentation of CVT have been proposed. ${ }^{(3)}$ Firstly, CVT can result in increased retrograde pressures in the cerebral capillaries and venules, leading to decreased cerebral perfusion, ischaemic injury and cytotoxic oedema. Disruption of the blood-brain barrier leads to vasogenic oedema, while rupture of capillaries and venules results in parenchymal haemorrhages. Secondly, CVT can result in impaired cerebrospinal fluid (CSF) absorption, as CSF is typically absorbed through arachnoid granulations into the superior sagittal sinus. This can lead to increased intracranial pressure.

Known risk factors for CVT include prothrombotic states, hormonal status (such as oral contraceptive use, pregnancy and puerperium), infection, chronic inflammatory disease, malignancy, haematological disorders and trauma. ${ }^{(1)}$ The recent COVID-19 pandemic has also brought considerable attention to the pro-thrombotic tendencies associated with the disease. ${ }^{(4)}$ There have been case reports of CVT associated with COVID-19. ${ }^{(5)}$ In our tropical climate, especially during the months of May to July, which are usually the hottest months in the year, dehydration is also a likely contributing factor, particularly affecting vulnerable groups, including elderly individuals who live alone and physically active young adults (such as soldiers). Clinicians and radiologists should have a high index of suspicion for CVT when approaching a case with these aforementioned risk factors.

\section{CEREBRAL VENOUS SYSTEM ANATOMY}

Fig. 1 shows the anatomy of the cerebral venous system. ${ }^{(6,7)}$

The dural venous sinuses are venous channels located between the dural layers forming the major drainage pathways of the brain. The superior sagittal sinus, inferior sagittal sinus and straight sinus converge at the torcular herophili, deep into the occipital protuberance. This drains into the bilateral transverse sinuses, sigmoid sinuses and internal jugular veins.

The superficial cerebral (cortical) veins are located in the subarachnoid space and drain into the nearest dural venous sinus, either directly or via anastomotic pathways. The vein of Trolard (superior anastomotic vein) connects the superficial middle cerebral veins with the superior sagittal sinus, while the vein of Labbé (inferior anastomotic vein) connects the superficial middle cerebral veins with the transverse sinus.

The deep venous system drains the thalami, septum pellucidum, basal ganglia, upper brainstem and deep white matter of the parietal, temporal and frontal lobes. The internal cerebral veins join the basal vein of Rosenthal to form the vein of Galen, which then joins the inferior sagittal sinus to form the straight sinus.

The superior sagittal sinus is the most commonly affected sinus in CVT (62.0\%), followed by the transverse and sigmoid sinuses $(41.2 \%-44.7 \%$ per side), straight sinus $(18.0 \%)$, cortical veins (17.1\%), jugular veins (11.9\%) and deep venous system (10.9\%). ${ }^{(1)}$

\section{IMAGING FINDINGS AND CASE DISCUSSION}

Imaging findings can be broadly divided into direct signs and indirect signs. ${ }^{(8)}$

One direct sign is a hyperdense thrombus seen on unenhanced head CT, which can be a cord-like hyperattenuating sinus classically seen in the transverse sinus on axial images (cord sign) or triangular dense vein that is typically seen in the posterior superior sagittal sinus (delta or pseudo-delta sign). Another direct sign is the loss of flow signal on unenhanced time-of-flight or phase-contrast magnetic resonance (MR) venography sequences. Direct visualisation of thrombus as a filling defect may be seen on contrast-enhanced CT/MR venography. A triangular filling defect within the superior sagittal sinus with surrounding enhancement on contrast-enhanced CT/MR venography can produce a classical 'empty delta sign'.

Indirect signs largely consist of parenchymal changes, which include vasogenic or cytotoxic white matter oedema, cortical swelling, changes in diffusion-weighted imaging/apparent diffusion coefficient values, cortical or meningeal enhancement and intracranial haemorrhage. ${ }^{(9,10)}$ Thrombosis may also be suspected when there is loss of normal venous flow void on routine T1-weighted (T1-W) and T2-weighted (T2-W) MR imaging sequences. 


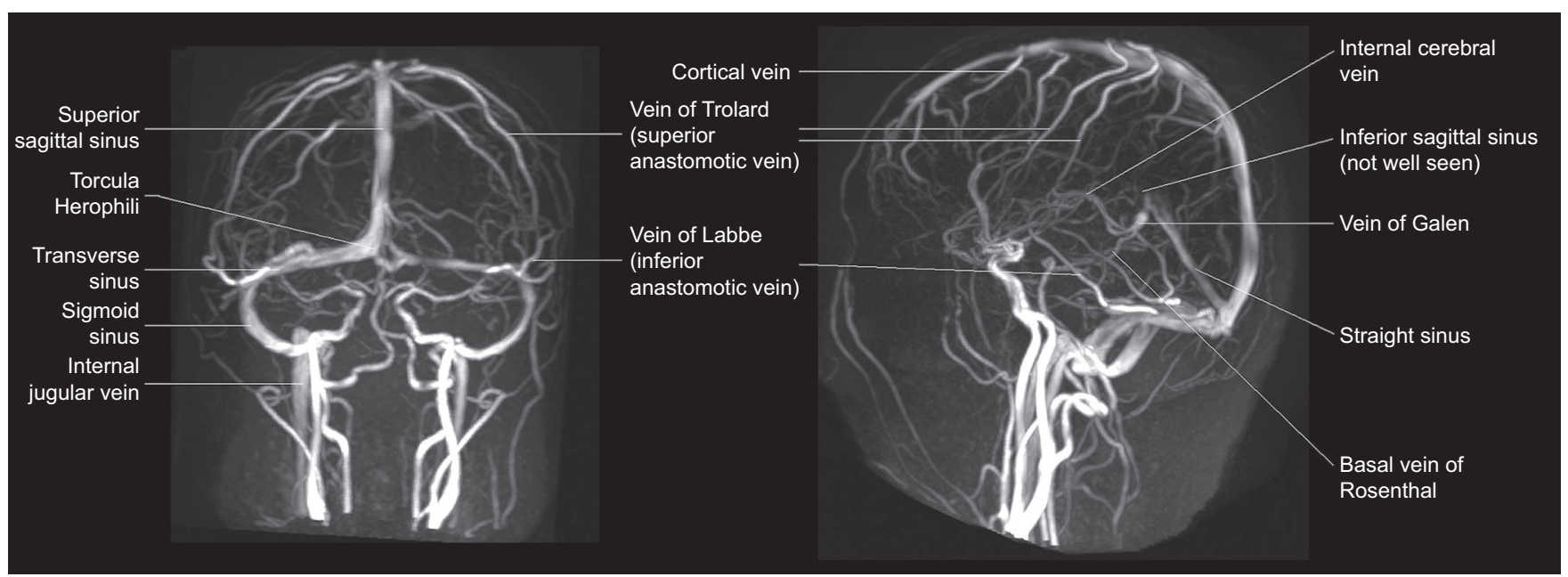

Fig. 1 Maximum-intensity projection reconstructions of three-dimensional phase-contrast magnetic resonance venograms in a 19-year-old woman show the anatomy of the cerebral venous system. Note the unilateral hypoplasia of the transverse sinus, a normal variant.
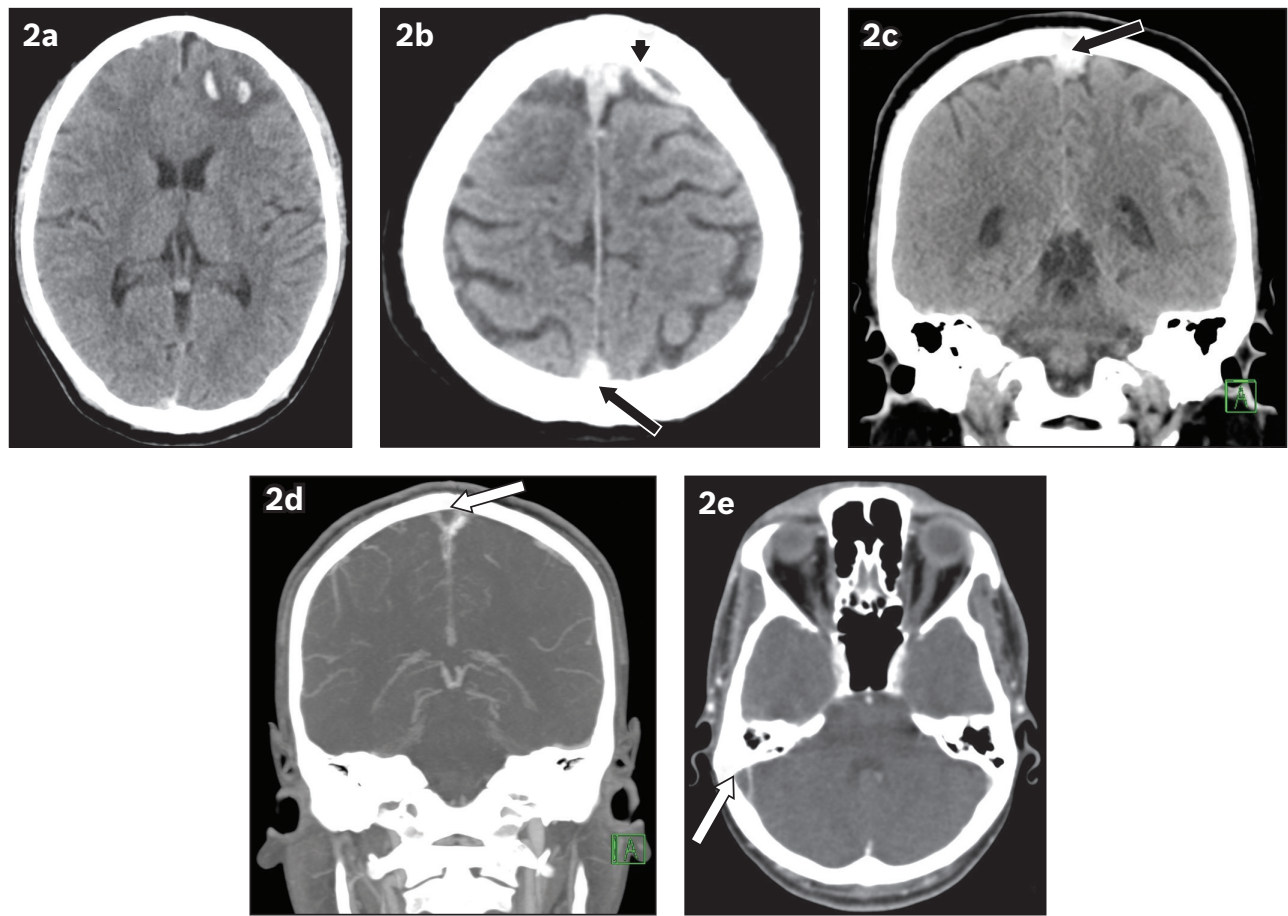

Fig. 2 A 27-year-old man presented with fall and seizure. (a) Unenhanced axial CT image of the head shows an intraparenchymal haematoma in the left frontal lobe. The case was initially managed as traumatic brain injury. (b \& c) On further review, the axial and coronal CT images show hyperdense cortical veins (arrowhead) and superior sagittal sinus (black arrows). (d \& e) Coronal and axial maximum-intensity projection reconstructions from a CT venogram confirm filling defects in the superior sagittal sinus, extending to the right transverse, sigmoid sinuses and right internal jugular vein (white arrows), with an 'empty delta' appearance.

Intraparenchymal haemorrhage as a complication of CVT may sometimes be misinterpreted as haemorrhagic contusions in patients with concurrent trauma (Fig. 2). Attention should always be paid to the dural venous sinuses on unenhanced head $\mathrm{CT}$, as a hyperdense acute thrombus may sometimes be seen within the cerebral venous sinuses (cord sign, Fig. 2). However, this finding has low sensitivity, with a reported frequency of $25 \%-56 \%$ in cases of confirmed CVT. ${ }^{(10-12)}$ In chronic thrombosis, the clot may return to normal attenuation and, hence, be difficult to detect.

Focal oedema on unenhanced CT may be mistaken for a mass lesion (Fig. 3). The presence of restricted diffusion in an atypical territory suggests cytotoxic oedema due to venous rather than arterial infarction. A good understanding of arterial and venous vascular territories and close attention to the appearance of the dural venous sinuses allows for a more confident diagnosis of CVT.

Isolated thrombosis involving the deep venous system may mimic other pathologies affecting the basal ganglia and thalami (Fig. 4). For example, differential diagnoses of bilateral thalamic lesions include arterial infarction (basilar tip or artery of Percheron), infections (such as Japanese encephalitis virus, toxoplasmosis), metabolic causes (such as Wernicke encephalopathy and Wilson's disease), central variant of posterior reversible encephalopathy syndrome and neoplasms. Deep CVT needs to be identified, as it is associated with a poorer prognosis. ${ }^{(1)}$

Dural sinus thrombosis can be chronic on presentation, with concurrent acute thrombosis in the cortical veins, as in our 

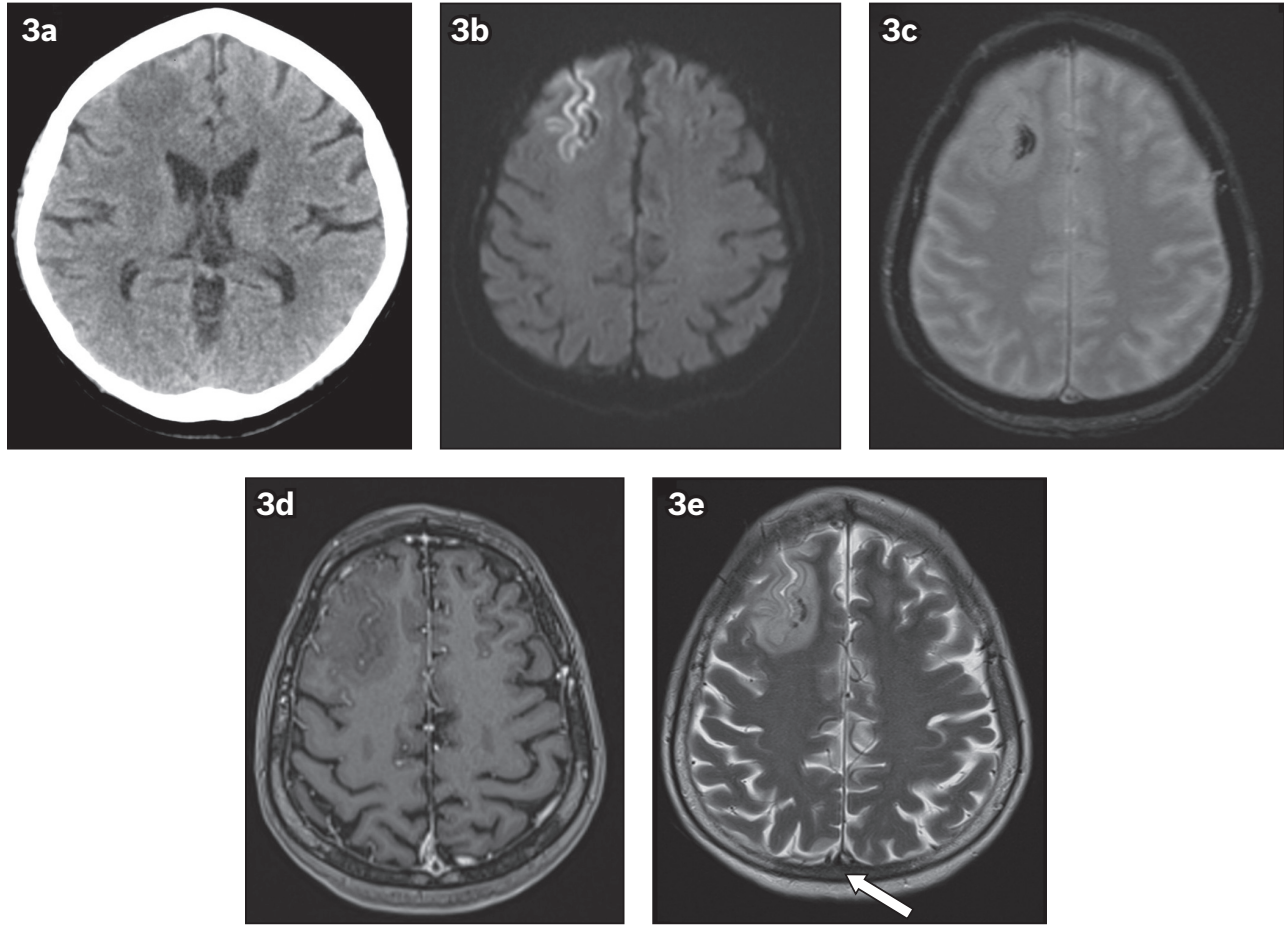

Fig. 3 A 51-year-old woman presented with seizures. (a) Unenhanced axial CT image of the head shows a focal hypodense lesion in the right frontal lobe, thought to be a mass. Indirect signs of venous infarction are noted, including (b) focal gyriform restricted diffusion on axial diffusion-weighted MR image; (c) associated small amount of haemorrhage on axial T2-W MR image; and (d) gyriform enhancement on contrast-enhanced axial T1-W MR image. (e) Axial T2-W MR image shows loss of flow void in the superior sagittal sinus, in keeping with thrombosis (arrow). This was further confirmed on a subsequent CT venogram (not shown). Further history-taking revealed use of oral contraceptives by the patient during her recent overseas travel.
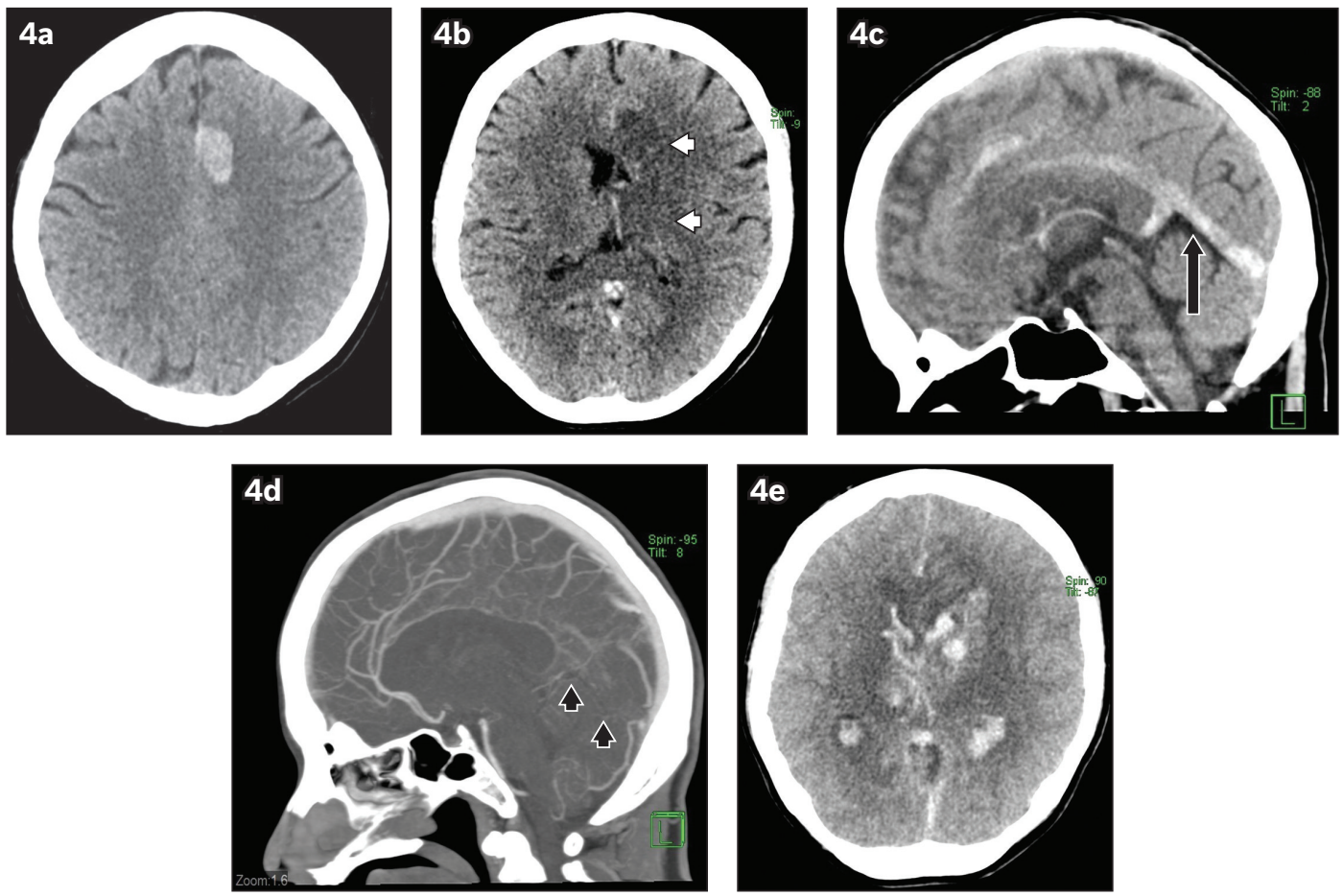

Fig. 4 A 41-year-old woman with a background of long-term oral contraceptive use for nine years presented with progressive drowsiness, giddiness, vomiting and generalised weakness. ( $a$ \& b) Unenhanced axial CT images of the head show an acute intraparenchymal haematoma in the parasagittal left frontal lobe as well as significant oedema in the basal ganglia and thalami (white arrowheads). (c) Sagittal CT image shows hyperdensity within the inferior sagittal sinus, vein of Galen and straight sinus (arrow). (d) Sagittal maximum-intensity projection reconstruction from CT venography shows loss of contrast opacification in the deep venous system, inferior sagittal and straight sinuses (black arrowheads). (e) A repeat CT image of the brain obtained a few days later shows worsening haemorrhage in the affected regions, diffuse subarachnoid and intraventricular haemorrhage as well as worsening mass effect, subsequently leading to the patient's death. 

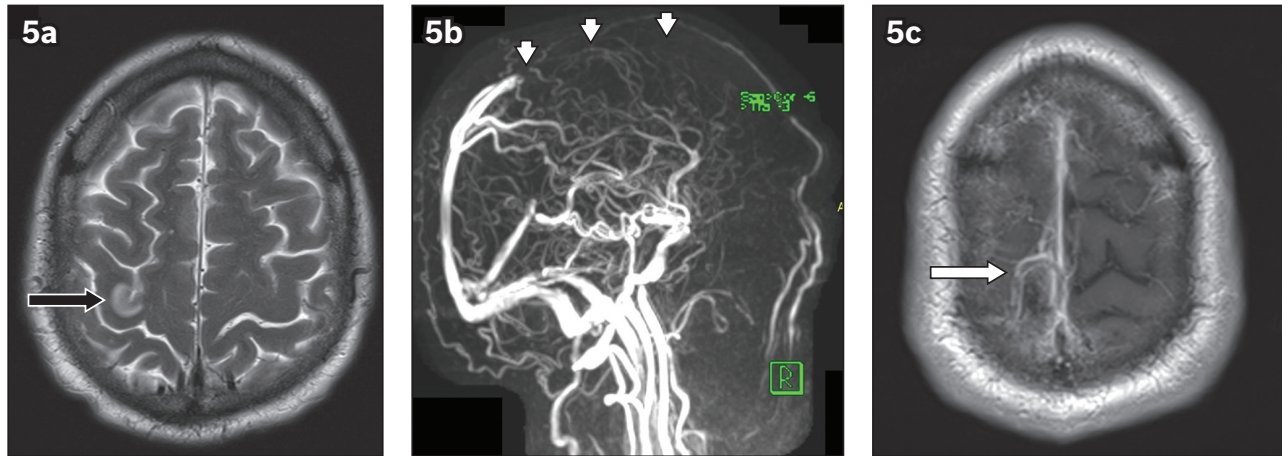

Fig. 5 A 62-year-old man presented with seizures. (a) Axial T2-W MR image shows focal oedema in the right perirolandic cortex (black arrow). (b) Maximumintensity projection reconstruction from three-dimensional phase-contrast MR venography subsequently shows loss of flow signal in the anterior twothirds of the superior sagittal sinus (arrowheads). (c) Contrast-enhanced axial T1-W MR image shows thin enhancement of the superior sagittal sinus, suggesting chronic thrombosis. A filling defect is also seen within a cortical vein at the vertex (white arrow), in keeping with acute thrombosis.
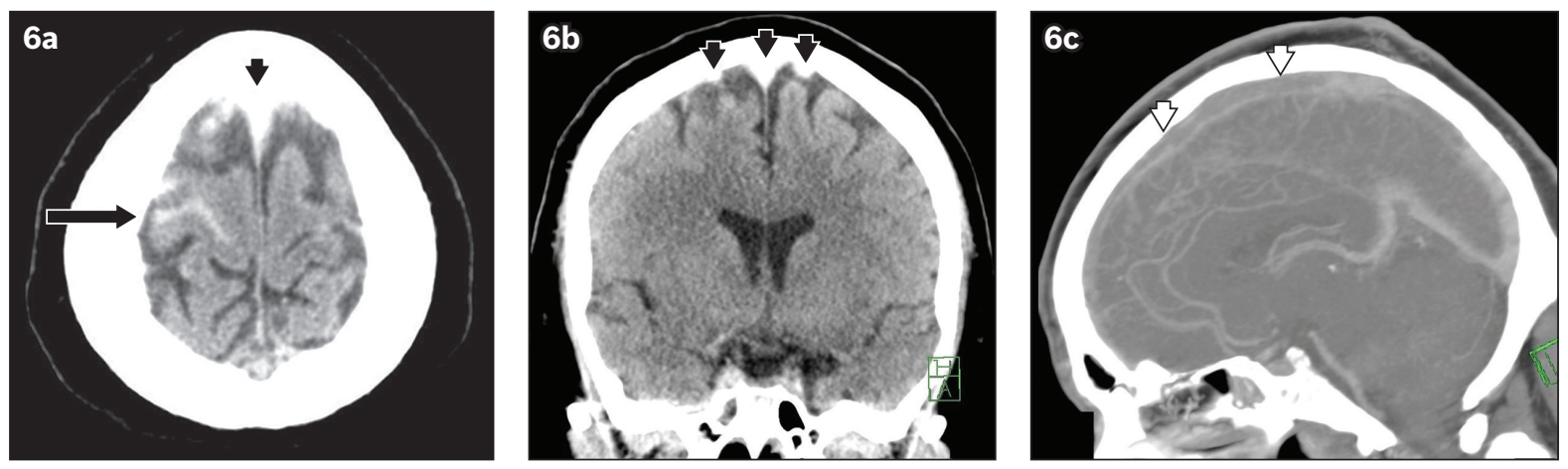

Fig. 6 A 35-year-old man presented with syncope. (a) Unenhanced axial CT image of the head shows right frontal acute subarachnoid haemorrhage (arrow). (a \& b) Unenhanced axial and coronal CT images show hyperdensity along the superior sagittal sinus and bilateral frontal cortical veins (black arrowheads). (c) Sagittal maximum intensity projection reconstruction from CT venography confirms filling defect in the anterior two thirds of the superior sagittal sinus (white arrowheads).
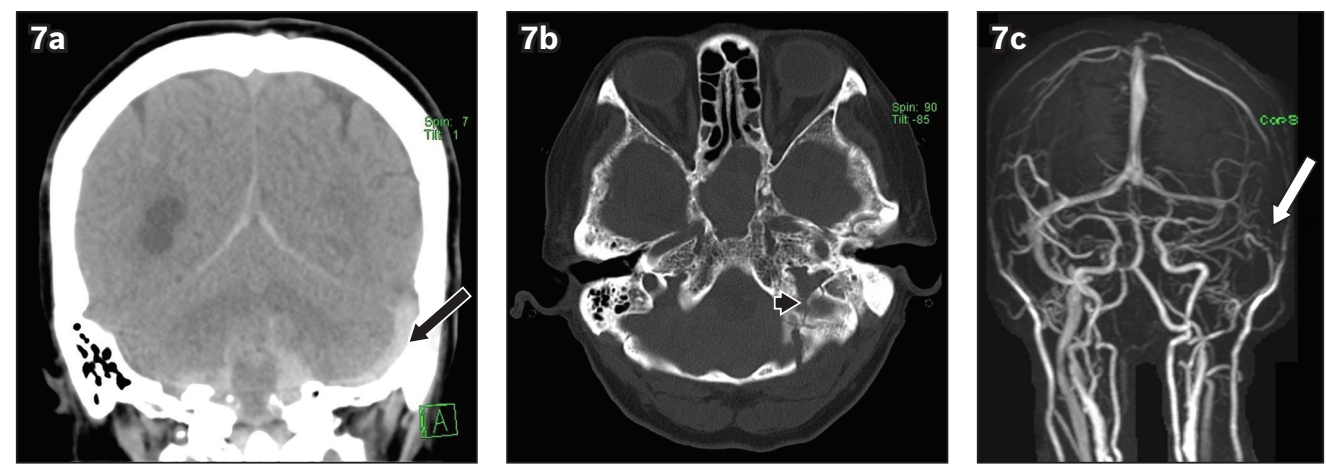

Fig. 7 A 60-year-old woman presented after a fall. (a) Unenhanced coronal head CT image shows subarachnoid haemorrhage, subdural haemorrhage and hyperdense left sigmoid sinus (black arrow). (b) Unenhanced axial head CT image with reconstructed bone window shows an undisplaced fracture in the left occipital and temporal bone extending into the left jugular foramen (arrowhead). (c) Maximum intensity projection reconstruction from threedimensional phase-contrast MR venogram shows loss of flow signal in the left sigmoid sinus (white arrow)

case (Fig. 5). Chronic thrombi have significant variability in MR signal intensity and appearance on conventional unenhanced MR venography techniques. These may appear as peripheral enhancement with central filling defect, irregular central and peripheral filling defects with heterogeneous enhancement or central enhancing channel on contrast-enhanced MR venography. ${ }^{(13)}$ Contrast-enhanced MR venography techniques are shown to be superior compared to non-contrast MR techniques such as threedimensional phase-contrast and two-dimensional time-of-flight. ${ }^{(14,15)}$

Rarely, CVT can result in acute subarachnoid haemorrhage (Fig. 6) ${ }^{(8)}$ which can be confused for other aetiologies in patients presenting with headache or trauma. Radiologists should pay special attention to dural venous sinuses in cases of convexal distribution of subarachnoid haemorrhage.

Trauma may also be a direct cause of CVT. The dural venous sinuses must be closely interrogated when there are fractures extending to or closely associated with them (Fig. 7). The diagnosis may be confounded by intracranial haemorrhage in the rest of the brain.

Long-term sequelae of CVT include dural arteriovenous fistula formation (Fig. 8) and increased intracranial pressure.

Various conditions and variants can render the interpretation of CVT difficult. ${ }^{(10)}$ Increased attenuation of dural sinuses on 

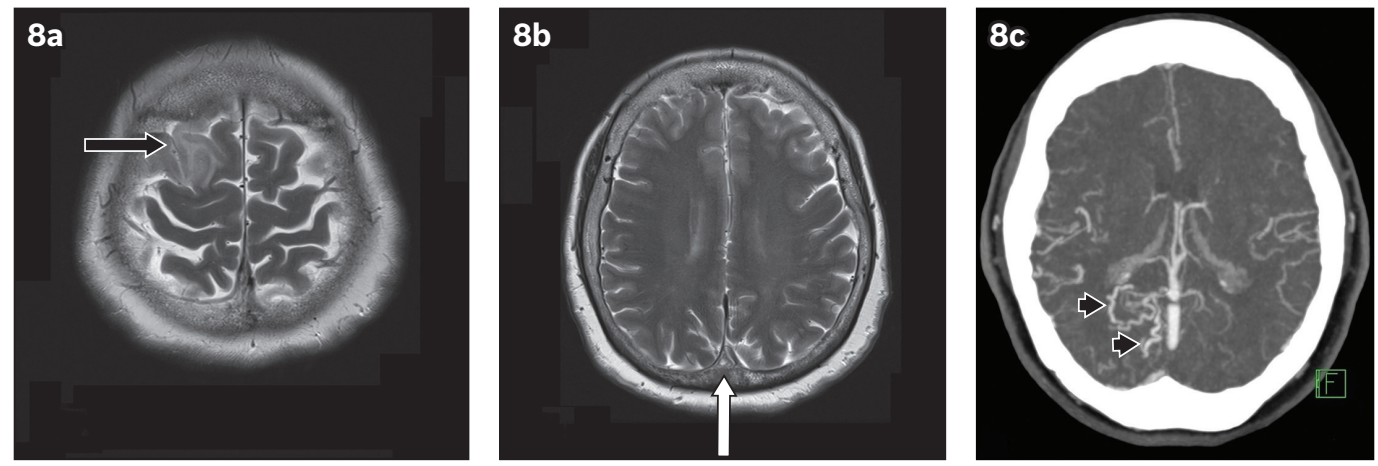

Fig. 8 A 66-year-old woman presented with non-ST-elevation myocardial infarction and had convulsive episodes during hospitalisation. (a \& b) Axial T2-W MR images show focal oedema in the right frontal lobe (black arrow) and loss of flow void in the superior sagittal sinus (white arrow). (c) Axial maximum-intensity projection reconstruction from a follow-up CT venogram eight months later shows persistent thrombosis in the superior sagittal sinus with abnormal dilated vessels adjacent to the sinus (arrowheads), indicating dural arteriovenous fistula formation.

unenhanced CT may be attributed to dehydration or high haematocrit values, in which case comparison with the blood pool of the other sinuses and arteries in the brain is imperative. Subdural or subarachnoid blood products may be mistaken for a hyperattenuating dural sinus owing to their close proximity. Hypoplasia or aplasia of dural sinuses, commonly seen unilaterally in the transverse sinus, may mimic loss of flow signal on unenhanced MR venography, in which case contrast-enhanced CT/MR techniques may be helpful. Arachnoid granulations can invaginate into dural sinuses and be mistaken for filling defects, although they typically have rounded shape, CSF density/signal and characteristic locations.

\section{CONCLUSION}

CVT, while uncommon, is associated with high morbidity and mortality if left untreated. It can be difficult to diagnose initially because of its non-specific clinical features and indirect imaging features, which may be misinterpreted. In the current COVID-19 pandemic, with increasing concerns about its thrombotic complications, clinicians and radiologists should be vigilant when approaching cases in their daily practice. This case series illustrates a wide range of possible imaging findings of CVT.

\section{REFERENCES}

1. Ferro JM, Canhão P, Stam J, Bousser MG, Barinagarrementeria F; ISCVT Investigators. Prognosis of cerebral vein and dural sinus thrombosis: results of the International Study on Cerebral Vein and Dural Sinus Thrombosis (ISCVT).
Stroke 2004; 35:664-70

2. Medel R, Monteith SJ, Crowley RW, Dumont AS. A review of therapeutic strategies for the management of cerebral venous sinus thrombosis. Neurosurg Focus 2009; 27:E6.

3. Stam J. Thrombosis of the cerebral veins and sinuses. N Engl J Med 2005; 352:1791-8

4. Klok FA, Kruip MJHA, van der Meer NJM, et al. Confirmation of the high cumulative incidence of thrombotic complications in critically ill ICU patients with COVID-19: an updated analysis. Thromb Res 2020; 191:148-50.

5. Cavalcanti DD, Raz E, Shapiro M, et al. Cerebral venous thrombosis associated with COVID-19. AJNR Am J Neuroradiol 2020; 41:1370-6.

6. Ayanzen RH, Bird CR, Keller PJ, et al. Cerebral MR venography: normal anatomy and potential diagnostic pitfalls. AJNR Am J Neuroradiol 2000; 21:74-8.

7. Farb RI, Scott JN, Willinsky RA, et al. Intracranial venous system: gadoliniumenhanced three-dimensional MR venography with auto-triggered elliptic centric-ordered sequence--initial experience. Radiology 2003; 226:203-9.

8. Linn J, Brückmann H. Cerebral venous and dural sinus thrombosis: state-of-theart imaging. Clin Neuroradiol 2010; 20:25-37.

9. Leach JL, Fortuna RB, Jones BV, Gaskill-Shipley MF. Imaging of cerebral venous thrombosis: current techniques, spectrum of findings, and diagnostic pitfalls. Radiographics 2006; 26 Suppl 1:S19-41; discussion S42-3.

10. Canedo-Antelo M, Baleato-González S, Mosqueira AJ, et al. Radiologic clues to cerebral venous thrombosis. Radiographics 2019; 39:1611-28.

11. Virapongse C, Cazenave C, Quisling R, Sarwar M, Hunter S. The empty delta sign: frequency and significance in 76 cases of dural sinus thrombosis. Radiology 1987; 162:779-85.

12. Teasdale E. Cerebral venous thrombosis: making the most of imaging. J R Soc Med 2000; 93:234-7.

13. Leach JL, Wolujewicz M, Strub WM. Partially recanalized chronic dural sinus thrombosis: findings on MR imaging, time-of-flight MR venography, and contrastenhanced MR venography. AJNR Am J Neuroradiol 2007; 28:782-9.

14. Liang L, Korogi $Y$, Sugahara T, et al. Evaluation of the intracranial dural sinuses with a $3 \mathrm{D}$ contrast-enhanced MP-RAGE sequence: prospective comparison with 2D-TOF MR venography and digital subtraction angiography. AJNR Am J Neuroradiol 2001; 22:481-92.

15. Lettau M, Laible M, Barrows RJ, et al. 3-T contrast-enhanced MR angiography with parallel imaging in cerebral venous and sinus thrombosis. J Neuroradiol $2011 ; 38: 275-82$ 


\section{SINGAPORE MEDICAL COUNCIL CATEGORY 3B CME PROGRAMME} (Code SMJ 202112B)

Question 1. Regarding cerebral venous thrombosis:

(a) The most common clinical presentation is seizure.

(b) The most common dural venous sinus involved is the superior sagittal sinus.

(c) The vein of Galen usually drains directly into the cavernous sinuses.

(d) Head trauma is a risk factor.

Question 2. The following are indirect signs of cerebral venous thrombosis:
(a) Restricted diffusion
(b) Vasogenic white matter oedema
(c) Filling defect within a sinus on contrast-enhanced magnetic resonance (MR) venography
(d) Cortical enhancement

Question 3. The following are direct signs of cerebral venous thrombosis:
(a) Cord sign
(b) Empty delta sign
(c) Intracranial haemorrhage
(d) Cerebral infarction

Question 4. Regarding cerebral venous thrombosis:

(a) Fractures extending to the dural venous sinuses can precipitate cerebral venous thrombosis.

(b) Cerebral venous thrombosis can present with acute convexal subarachnoid haemorrhage.

(c) A hyperdense thrombus seen on unenhanced computed tomography is a highly sensitive (> 90\%) finding for cerebral venous thrombosis.

(d) Deep cerebral venous thrombosis has a better prognosis than thrombosis of the superficial dural venous sinuses.

Question 5. Regarding imaging of cerebral venous thrombosis:

(a) Japanese encephalitis with bilateral thalamic haemorrhagic lesions can mimic deep cerebral venous thrombosis.

(b) Two-dimensional time-of-flight MR venography is a contrast-enhanced technique.

(c) Unenhanced MR venography techniques are superior to contrast-enhanced MR venography in evaluating chronic cerebral venous thrombosis.

(d) One should compare the attenuation of the dural venous sinuses to the rest of the intracranial blood pool before deciding that it is hyperdense.

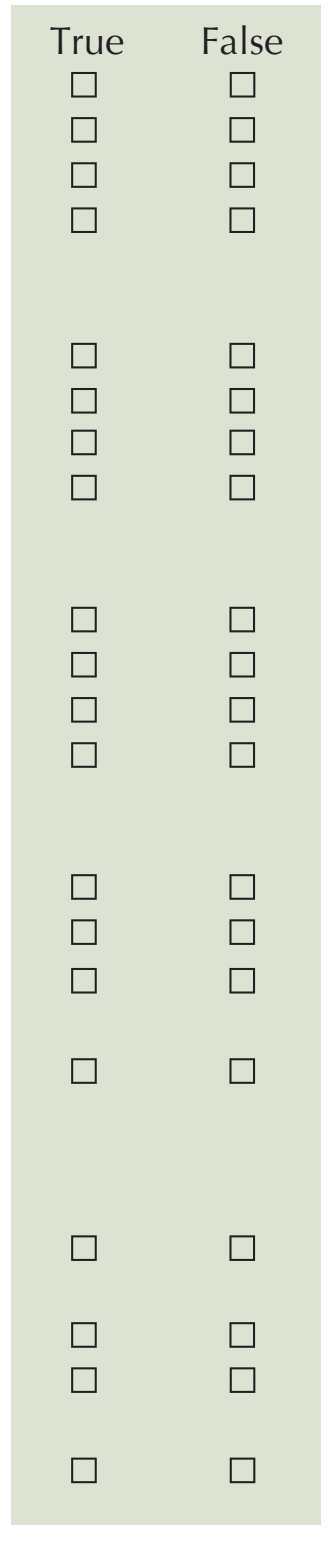

\section{Doctor's particulars:}

Name in full:

MCR no.:

Specialty:

Email:

\section{SUBMISSION INSTRUCTIONS}

Visit the SMJ website: http://www.smj.org.sg/current-issue and select the appropriate quiz. You will be redirected to the SMA login page.

For SMA member: (1) Log in with your username and password (if you do not know your password, please click on 'Forgot your password?'). (2) Select your answers for each quiz and click 'Submit'.

For non-SMA member: (1) Create an SMJ CME account, or log in with your SMJ CME username and password (for returning users). (2) Make payment of SGD 21.40 (inclusive of $7 \%$ GST) via PayPal to access this month's quizzes. (3) Select your answers for each quiz and click 'Submit'.

RESULTS:

(1) Answers will be published online in the SMJ February 2022 issue. (2) The MCR numbers of successful candidates will be posted online at the SMJ website by 24 February 2022. (3) Passing mark is $60 \%$. No mark will be deducted for incorrect answers. (4) The SMJ editorial office will submit the list of successful candidates to the Singapore Medical Council. (5) One CME point is awarded for successful candidates. (6) SMC credits CME points according to the month of publication of the CME article (i.e. points awarded for a quiz published in the December 2021 issue will be credited for the month of November 2021, even if the deadline is in February 2022).

Deadline for submission (December 2021 SMJ 3B CME programme): 12 noon, 17 February 2022. 